\title{
Radiotherapy Dose-Volume Effects on Salivary Gland Function
}

\author{
Joseph O. Deasy, Ph.D. ${ }^{*}$ Vitali Moiseenko, Ph.D. ${ }^{\dagger}$, Lawrence Marks, M.D. ${ }^{\ddagger}$, K. S. Clifford \\ Chao, M.D.§, Jiho Nam, Ph.D. $\ddagger$, and Avraham Eilsbruch, M.D.ף \\ *Department of Radiation Oncology, Washington University School of Medicine and Alvin J. \\ Siteman Cancer Center, St. Louis, MO \\ ${ }^{\dagger}$ Department of Medical Physics, British Columbia Cancer Agency-Vancouver Cancer Center, \\ Vancouver, BC, Canada \\ FLineberger Comprehensive Cancer Center, University of North Carolina, Chapel Hill, NC \\ §Department of Radiation Oncology, Columbia School of Medicine, New York, NY \\ "Department of Radiation Oncology, University of Michigan School of Medicine, Ann Arbor, MI
}

\begin{abstract}
Publications relating parotid dose-volume characteristics to radiotherapy-induced salivary toxicity were reviewed. Late salivary dysfunction has been correlated to the mean parotid gland dose, with recovery occurring with time. Severe xerostomia (defined as long-term salivary function of $<25 \%$ of baseline) is usually avoided if at least one parotid gland is spared to a mean dose of less than $\approx 20$ Gy or if both glands are spared to less than $\approx 25 \mathrm{~Gy}$ (mean dose). For complex, partialvolume RT patterns (e.g., intensity-modulated radiotherapy), each parotid mean dose should be kept as low as possible, consistent with the desired clinical target volume coverage. A lower parotid mean dose usually results in better function. Submandibular gland sparing also significantly decreases the risk of xerostomia. The currently available predictive models are imprecise, and additional study is required to identify more accurate models of xerostomia risk.
\end{abstract}

\section{Keywords}

Xerostomia; salivary parotid glands; submandibular salivary glands; radiotherapy; dose-volume effects

\section{Clinical Significance}

Radiotherapy (RT) is commonly used to treat head-and-neck tumors. In these treatments, the parotid, submandibular, and minor salivary glands are often incidentally irradiated. A reduction in salivary function is a common toxicity and reduces the patient's quality of life (QOL). Inadequate salivary function (“xerostomia") leads to multiple problems, including

Copyright $\odot 2010$ Elsevier Inc.

Reprint requests to: Joseph O. Deasy, Ph.D., Department of Radiation Oncology, Washington University School of Medicine, 4921 Parkview P1., St. Louis, MO 63110. Tel: (314) 362-1420; Fax: (314) 362-8521; jdeasy @ radonc.wustl.edu.

Conflict of interest: none. 
poor dental hygiene, a propensity to oral infections, sleep disturbances, oral pain, and difficulty chewing and swallowing. Stimulated salivary production is largely (60-70\% of total) derived from the parotid glands, with the balance from other glands. Resting (unstimulated) salivary production is due primarily to the submandibular and sublingual glands and numerous small oral salivary glands (1).

\section{Endpoints}

Xerostomia can be defined according to the patient's symptoms (e.g., altered taste or sensations of dryness) or quantified saliva production. Objective criteria include measured salivary production at rest or stimulation and imaging endpoints. Observer-based, Common Terminology Criteria for Adverse Events, include the requirement for frequent drinks of water or diet alterations. Imaging endpoints include scintigraphy of parotid gland ejection fraction over a timed interval (2) and dynamic magnetic resonance imaging sialography of ductal flow (3).

Salivary function is quantifiable. Grade 4 xerostomia is defined by the Late Effects Normal Tissue-Subjective, Objective, Management, Analytic (LENT-SOMA) scale as an objective reduction of $\geq 75 \%$ of baseline salivary function. Whole mouth salivary function is typically assessed by asking the subject to produce as much saliva as possible within a given period (often $5 \mathrm{~min}$ ). This can be performed in an unstimulated (at rest) or stimulated manner (in response to a salivary stimulant). However, salivary function measurements are uncertain and variable, with standard deviations of $\approx 20-30 \%$ reported for whole mouth measurements (4).

The patient-reported outcomes/QOL instruments used have included xerostomia-specific forms (5). Correlations between salivary flow and QOL have been inconsistent. Observerbased monitoring of xerostomia symptoms can underestimate the actual xerostomia symptoms compared with patient-reported symptoms (5). Because many reports of xerostomia after intensity-modulated RT have relied on observer-rated scores, the overall severity of xerostomia in recently published data might have been underestimated.

\section{Damage Recovery}

A reduction in salivary function can begin within 1 week of the initiation of RT and usually persists afterward. Function often gradually recovers within $\approx 2$ years after RT (unless the radiation damage is too severe) $(6,7)$. Moreover, recovery overshoot (i.e., recovery to $>100 \%)$ in salivary function can occur $(8,9)$. Braam et al. (10) reported that recovery in parotid flow correlated significantly with a reduction in patient-reported dry mouth symptoms.

\section{Challenges in Defining Volumes}

Parotid and submandibular salivary glands can be adequately delineated on contrastenhanced computed tomography images. However, irradiated parotid glands typically shrink during RT, presumably owing to cell loss. On the basis of weekly computed tomography scans of 15 patients, Robar et al. (11) reported little change in the medial parotid gland 
position during RT. However, the lateral edges shrank, on average, $\approx 1 \mathrm{~mm} / \mathrm{wk}$ during RT (average displacements of 4-6 mm during the RT course), resulting in decreasing gland sparing

\section{Review of Dose-Volume Data}

A variety of salivary endpoints have been correlated with the dosimetric dose-volume parameters, including subjective xerostomia and objective stimulated/unstimulated salivary flow. In particular, the mean parotid gland dose $(6,8,9,11)$ has been correlated with whole mouth or individual gland salivary production. Table 1 summarizes the reported dosevolume predictors for salivary flow, the incidence of complications, and salivary function recovery.

Minimal gland function reduction occurs at $<10-15$ Gy mean dose. Gland function reduction gradually increases at radiation doses of $20-40 \mathrm{~Gy}$, with a strong reduction (usually by $>75 \%$ ) at $>40$ Gy (Fig. 1) $(4,6)$. Xerostomia risk is reduced with sparing of at least one parotid gland or even one submandibular gland (12). In one study, patients receiving <30 Gy to the contralateral parotid reported no or mild subjective xerostomia (13).

Some recovery of function occurs with time, with the tissue dose required for a 50\% response (TD50) increasing (i.e., more dose needed for the same level of injury) at longer follow-up times (Fig. 2) (8, 10, 12, 14-16). Figure 3 summarizes the existing published data regarding TD50 (dose resulting in 50\% incidence) for a reduction in stimulated saliva by $50-75 \%(2,6,10,14-20)$. Whole mouth or ipsilateral salivary measurement-based TD50s tend to be lower than scintigraphy-based TD50s ( $\approx 25-45$ Gy). Consistent with this, the image-based data shown in Fig. 4 implies a greater TD50 compared with the salivary flow data in Fig. 1. The wide variation in the reported TD50 values is unexplained but could result from several factors, including differences in dose distributions, salivary measurement methods, segmentation, intragland sensitivity, and so forth.

\section{Factors Affecting Risk}

Non-dose-volume factors could affect the risk of xerostomia. Nondosimetric patient factors (e.g., gender and age) and the use of chemotherapy have typically not correlated with xerostomia risk. However, pretreatment salivary function and medications affecting salivary function can affect the risk of xerostomia.

\section{Mathematical Modeling}

Several investigators have tried to fit dose, volume, and complication risk data to a sigmoidal response function. This mirrors the local function curve derived from imaging measurements (Fig. 4). The Lyman-Kutcher-Burman volume effect parameter, n, is typically set to 1 , although the best-fit value of $n$ has sometimes been reported to be either less (6) or greater (14) than 1. Chao et al.(4) fit stimulated whole mouth salivary function to a sum of two exponentials, representing contributions from both glands. Single gland function at 6 months was approximately given by $\exp (-0.054 \times$ mean gland dose $)(2)$. This model neglects the known submandibular gland contributions, however, which might cause it to 
overestimate the reduction at low mean doses (mean dose, $<15 \mathrm{~Gy}$ ). More complicated models have shown only minor improvements (6). Consistent with this, patient-specific flow predictions using mean gland doses have significant uncertainties (21). The function of the parotid glands should be modeled separately, because the glands seem to respond independently (Fig. 1).

\section{Fraction size effects on salivary function}

Detailed studies addressing fractionation have been limited, with conflicting results. In rats, Franzen et al.(22) estimated the $\alpha / \beta$ ratio for early effects to be high, $\approx 20 \mathrm{~Gy}$. The clinical Continuous hyperfractionated accelerated radiotherapy (CHART) experience has suggested that hyperfractionation has a protective effect on late function, consistent with a low $\alpha / \beta$ ratio (23). However, in rhesus monkeys, acini cell number reduction at 16 weeks after RT was worse for CHART hyperfractionation than for conventional fractionation to a similar dose (24), consistent with a relatively high $\alpha / \beta$ ratio. Thus, it might be that acute effects have a high $\alpha / \beta$ ratio and late damage (dependent on stem cell recovery [25]) has a low $\alpha / \beta$ ratio.

\section{Possible intragland sensitivity variations}

Function and response are typically assumed to be uniform throughout the parotid gland. However, this might not be accurate. In rats (26), RT to the cranial half produced more functional loss than RT to the caudal half. This finding relates to the specific anatomy of the rat parotid gland, in which the saliva from the caudal part flows through the cranial part and is therefore affected by damage to the cranial ductules. Although human parotid gland anatomy is more complex, it is possible that similar phenomena occur in humans, and this might be the cause of some of the variation in the reported mean dose TD50 values.

\section{Special Situations}

Submandibular gland sparing can reduce the risk of both stimulated and unstimulated xerostomia (27). Jellema et al. (28) reported that the mean dose to the parotid glands and the mean dose to the submandibular glands were both selected on multivariate analysis for patient-reported xerostomia. A hypothetical patient receiving a mean dose of $30 \mathrm{~Gy}$ to the parotid glands was estimated to have the risk of xerostomia decrease by $\approx 20 \%$ if the mean dose to the submandibular gland was 0 Gy compared with $50 \mathrm{~Gy}$. Similarly, surgical transfer of the submandibular gland out of the high-dose radiation field was reported to spare $\approx 30 \%$ of pretreatment stimulated salivary function (29). Regarding the dose response, MurdochKinch et al. (30) reported that submandibular gland-stimulated salivary function decreased significantly after a mean dose of $>40 \mathrm{~Gy}$. It is, therefore, not clear whether the parotid and submandibular glands have the same dose-volume response characteristics.

The mean dose to the oral cavity (containing minor salivary glands) has been found to be an independent risk factor in some data sets (7) but not others (25), probably because of technique differences.

The chemical modifier amifostine, is a radioprotector and has recently been shown to reduce the rates of xerostomia. Although quantitative data are sparse, Munter et al. (31) noted that amifostine significantly increased the combined parotid and submandibular gland tolerance 
dose for scintigraphically measured clearance dysfunction, by a mean dose of approximately 9 Gy.

\section{Recommended Dose-Volume Limits}

Sparing at least one parotid gland appears to eliminate xerostomia (Fig. 1), and sparing at least one submandibular gland also appears to reduce xerostomia risk and increase stimulated and unstimulated salivary function. Some of the reduction in stimulated salivary function in Fig. 1 also resulted from consistently irradiated submandibular glands. Severe xerostomia (long-term salivary function $<25 \%$ of baseline) can usually be avoided if at least one parotid gland has been spared to a mean dose of less than $\approx 20$ Gy or if both glands have been spared to a mean dose of less than $\approx 25 \mathrm{~Gy}$.

For complex partial volume RT patterns (e.g., intensity-modulated RT), the mean dose to each parotid gland should be kept as low as possible, consistent with the desired clinical target volume coverage. A lower mean dose to the parotid gland usually results in better function, even for relatively low mean doses ( $<10 \mathrm{~Gy})$. Similarly, the mean dose to the parotid gland should still be minimized, consistent with adequate target coverage, even if one or both cannot be kept to a threshold of $<20$ or $<25 \mathrm{~Gy}$. Published variations in response among different patient cohorts were probably related to the lack of an accurate model that correctly includes the effects of multiple salivary glands and intragland sensitivity variations. When it can be deemed oncologically safe, submandibular gland sparing to modest mean doses ( $<35$ Gy to see any effect) might reduce xerostomia symptoms.

\section{Future Toxicity Studies}

To improve patient-specific predictions, several questions need additional research:

1. Whether partial sparing (achievable with intensity-modulated RT) of the submandibular glands or minor glands within the oral cavity will have a positive effect on patients' QOL

2. Whether the (arbitrary) 25\% salivary threshold is the best quantitative measure with respect to the affects on patient QOL

3. Whether spatial/anatomic variations exist in the local radiation effect

4. Whether parotid gland shrinkage during RT should be explicitly accounted for in functional predictions

5. How submandibular sparing should be incorporated into predictive salivary function models

6. The quantitative effect on xerostomia of oral cavity sparing

7. The effect of the radioprotector amifostine on whole mouth salivary function

8. The reason imaging endpoints result in greater TD50 values than direct salivary measurements 
An overarching goal is the validation of an accurate predictive salivary function model. This will probably require combining multiple institutional or cooperative group data sets.

\section{Toxicity Scoring}

To best define xerostomia, we recommend that an observer-based system (e.g., the Common Terminology Criteria for Adverse Events) be supplemented by a validated QOL measurement device (e.g., the XQ (xerostomia questionnaire) [7]) and/or salivary measurements (e.g., whole mouth-stimulated measurements).

\section{Acknowledgments}

Partially funded by National Institutes of Health Grant R01 CA85181 (to J. O. Deasy) and Grant CA69579 (L. B. Marks) and by the American Association of Medical Physicists and the American Society of Therapeutic Radiology.

\section{References}

1. Dawes C, Wood CM. The contribution of oral minor mucous gland secretions to the volume of whole saliva in man. Arch Oral Biol. 1973; 18:337-342. [PubMed: 4515961]

2. Buus S, Grau C, Munk O, et al. Individual radiation response of parotid glands investigated by dynamic 11C-methionine PET. Radiother Oncol. 2006; 78:262-269. [PubMed: 16545879]

3. Astreinidou E, Roesink JM, Raaijmakers CP, et al. 3D MR sialography as a tool to investigate radiation-induced xerostomia: Feasibility study. Int J Radiat Oncol Biol Phys. 2007; 68:1310-1319. [PubMed: 17482767]

4. Chao KSC, Deasy JO, Markman J, et al. A prospective study of salivary function sparing in patients with head-and-neck cancers receiving intensity-modulated or three-dimensional radiation therapy: Initial results. Int J Radiat Oncol Biol Phys. 2001; 49:907-916. [PubMed: 11240231]

5. Meirovitz A, Murdoch-Kinch C, Schipper M, et al. Grading xerostomia by physicians or by patients after intensity-modulated radiotherapy of head-and-neck cancer. Int J Radiat Oncol Biol Phys. 2006; 66:445-453. [PubMed: 16839705]

6. Blanco AI, Chao KSC, El Naqa I, et al. Dose-volume modeling of salivary function in patients with head-and-neck cancer receiving radiotherapy. Int J Radiat Oncol Biol Phys. 2005; 62:1055-1069. [PubMed: 15990009]

7. Eisbruch A, Kim KM, Terrell JE, et al. Xerostomia and its predictors following parotid-sparing irradiation of head-and-neck cancer. Int J Radiat Oncol Biol Phys. 2001; 50:695-704. [PubMed: 11395238]

8. Maes A, Weltens C, Flamen P, et al. Preservation of parotid function with uncomplicated conformal radiotherapy. Radiother Oncol. 2002; 63:203-211. [PubMed: 12063010]

9. Li Y, Taylor J, Ten Haken R, et al. The impact of dose on parotid salivary recovery in head and neck cancer patients treated with radiation therapy. Int J Radiat Oncol Biol Phys. 2007; 67:660-669. [PubMed: 17141973]

10. Braam PM, Roesink JM, Raaijmakers CP, et al. Quality of life and salivary output in patients with head-and-neck cancer five years after radiotherapy. Radiat Oncol. 2007; 2:3. [PubMed: 17207274]

11. Robar JL, Day A, Clancey J, et al. Spatial and dosimetric variability of organs at risk in head-andneck intensity-modulated radiotherapy. Int J Radiat Oncol Biol Phys. 2007; 68:1121-1130. [PubMed: 17398025]

12. Saarilahti K, Kouri M, Collan J, et al. Sparing of the submandibular glands by intensity modulated radiotherapy in the treatment of head and neck cancer. Radiother Oncol. 2006; 78:270-275. [PubMed: 16564589]

13. Portaluri M, Fucilli F, Castagna R, et al. Three-dimensional conformal radiotherapy for locally advanced (stage II and worse) head-and-neck cancer: Dosimetric and clinical evaluation. Int J Radiat Oncol Biol Phys. 2006; 66:1036-1043. [PubMed: 16750321] 
14. Roesink JM, Moerland MA, Battermann JJ, et al. Quantitative dose-volume response analysis of changes in parotid gland function after radiotherapy in the head-and-neck region. Int J Radiat Oncol Biol Phys. 2001; 51:938-946. [PubMed: 11704314]

15. Roesink JM, Moerland MA, Hoekstra A. Scintigraphic assessment of early and late parotid gland function after radiotherapy for head-and-neck cancer: A prospective study of dose-volume response relationships. Int J Radiat Oncol Biol Phys. 2004; 58:1451-1460. [PubMed: 15050323]

16. Bussels B, Maes A, Flamen P, et al. Dose-response relationships within the parotid gland after radiotherapy for head and neck cancer. Radiother Oncol. 2004; 73:297-306. [PubMed: 15588874]

17. Eisbruch A, Ten Haken R, Kim H, et al. Dose, volume, and function relationships in parotid salivary glands following conformal and intensity-modulated irradiation of head and neck cancer. Int J Radiat Oncol Biol Phys. 1999; 45:577-587. [PubMed: 10524409]

18. Munter MW, Karger CP, Hoffner SG, et al. Evaluation of salivary gland function after treatment of head-and-neck tumors with intensity-modulated radiotherapy by quantitative pertechnetate scintigraphy. Int J Radiat Oncol Biol Phys. 2007; 58:175-184. [PubMed: 14697436]

19. Munter MW, Hoffner S, Hof H, et al. Changes in salivary gland function after radiotherapy of head and neck tumors measured by quantitative pertechnetate scintigraphy: Comparison of intensitymodulated radiotherapy and conventional radiation therapy with and without amifostine. Int $\mathbf{J}$ Radiat Oncol Biol Phys. 2007; 67:651-659. [PubMed: 17175118]

20. Rudat V, Munter M, Rades D, et al. The effect of amifostine or IMRT to preserve the parotid function after radiotherapy of the head and neck region measured by quantitative salivary gland scintigraphy. Radiother Oncol. 2008; 89:71-80. [PubMed: 18707782]

21. Deasy JO, Chao KSC, Markman J. Uncertainties in model-based outcome predictions for treatment planning. Int J Radiat Oncol Biol. 2001; 51:1389-1399.

22. Franzen L, Sundstrom S, Karlsson M, et al. Fractionated irradiation and early changes in noradrenaline induced potassium efflux $(86 \mathrm{Rb}+)$ in rat parotid gland. Acta Oncol. 1992; 31:359364. [PubMed: 1622658]

23. Leslie MD, Dische S. The early changes in salivary gland function during and after radiotherapy for head and neck cancer. Radiother Oncol. 1994; 30:26-32. [PubMed: 8153377]

24. Price RE, Ang KK, Stephens LC, et al. Effects of continuous hyperfractionated accelerated and conventionally fractionated radiotherapy on the parotid and submandibular salivary glands of rhesus monkeys. Radiother Oncol. 1995; 34:39-46. [PubMed: 7792397]

25. Lombaert IM, Brunsting JF, Wierenga PK, et al. Rescue of salivary gland function after stem cell transplantation in irradiated glands. PLoS ONE. 2008; 3:e2063. [PubMed: 18446241]

26. Konings AW, Cotteleer F, Faber H, et al. Volume effects and region-dependent radiosensitivity of the parotid gland. Int J Radiat Oncol Biol Phys. 2005; 62:1090-1095. [PubMed: 15990013]

27. Saarilahti K, Kouri M, Collan J, et al. Sparing of the submandibular glands by intensity modulated radiotherapy in the treatment of head and neck cancer. Radiother Oncol. 2006; 78:270-275. [PubMed: 16564589]

28. Jellema AP, Doornaert P, Slotman BJ, et al. Does radiation dose to the salivary glands and oral cavity predict patient-rated xerostomia and sticky saliva in head and neck cancer patients treated with curative radiotherapy? Radiother Oncol. 2005; 77:164-171. [PubMed: 16256229]

29. Seikaly H, Jha N, Harris JR, et al. Long-term outcomes of submandibular gland transfer for prevention of postradiation xerostomia. Arch Otolaryngol Head Neck Surg. 2004; 130:956-961. [PubMed: 15313866]

30. Murdoch-Kinch CA, Kim HM, Vineberg KA, et al. Dose-effect relationships for the submandibular salivary glands and implications for their sparing by intensity modulated radiotherapy. Int J Radiat Oncol Biol Phys. 2008; 72:373-382. [PubMed: 18337023]

31. Munter MW, Hoffner S, Hof H, et al. Changes in salivary gland function after radiotherapy of head and neck tumors measured by quantitative pertechnetate scintigraphy: Comparison of intensitymodulated radiotherapy and conventional radiation therapy with and without amifostine. Int $\mathbf{J}$ Radiat Oncol Biol Phys. 2007; 67:651-659. [PubMed: 17175118] 
(A) - 6 mos. post-RT

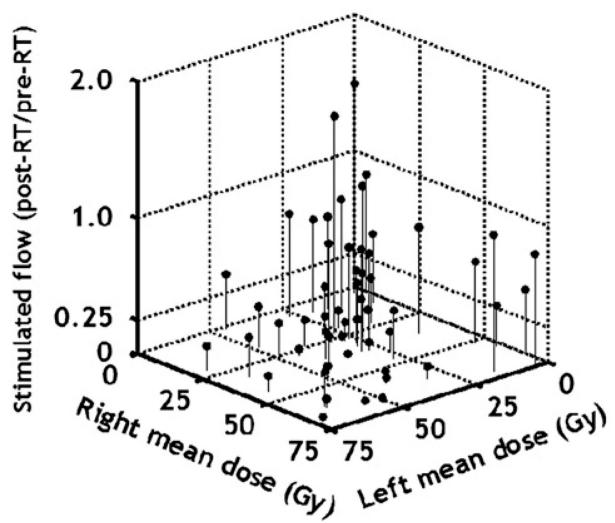

(B) - 12 mos. post-RT

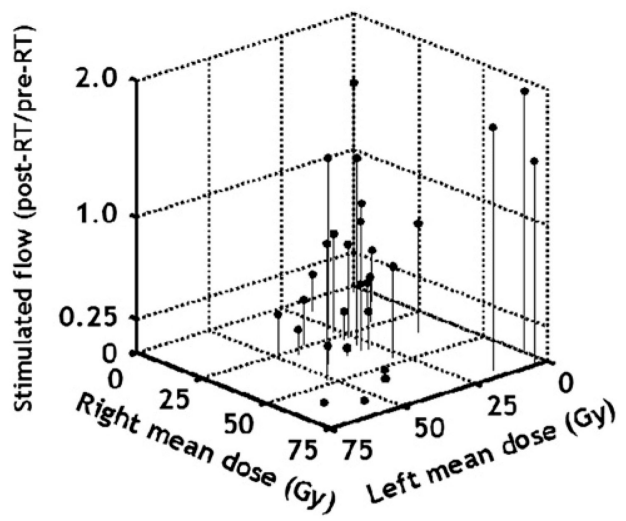

Fig. 1.

Stimulated whole mouth salivary measurements vs. mean parotid gland dose. Summary of Washington University stimulated salivary results at 6 and 12 months of follow-up. Data showed that when either gland was spared ( $<20$ Gy mean dose), ratio of post-radiotherapy (RT) to pre-RT flow is usually $>0.25$. Note, if either gland was highly spared ( $<10-15 \mathrm{~Gy})$, resulting salivary function will usually be high, regardless of irradiation level of the other parotid gland (data originally presented in Blanco et al. [6], but redrawn here). 


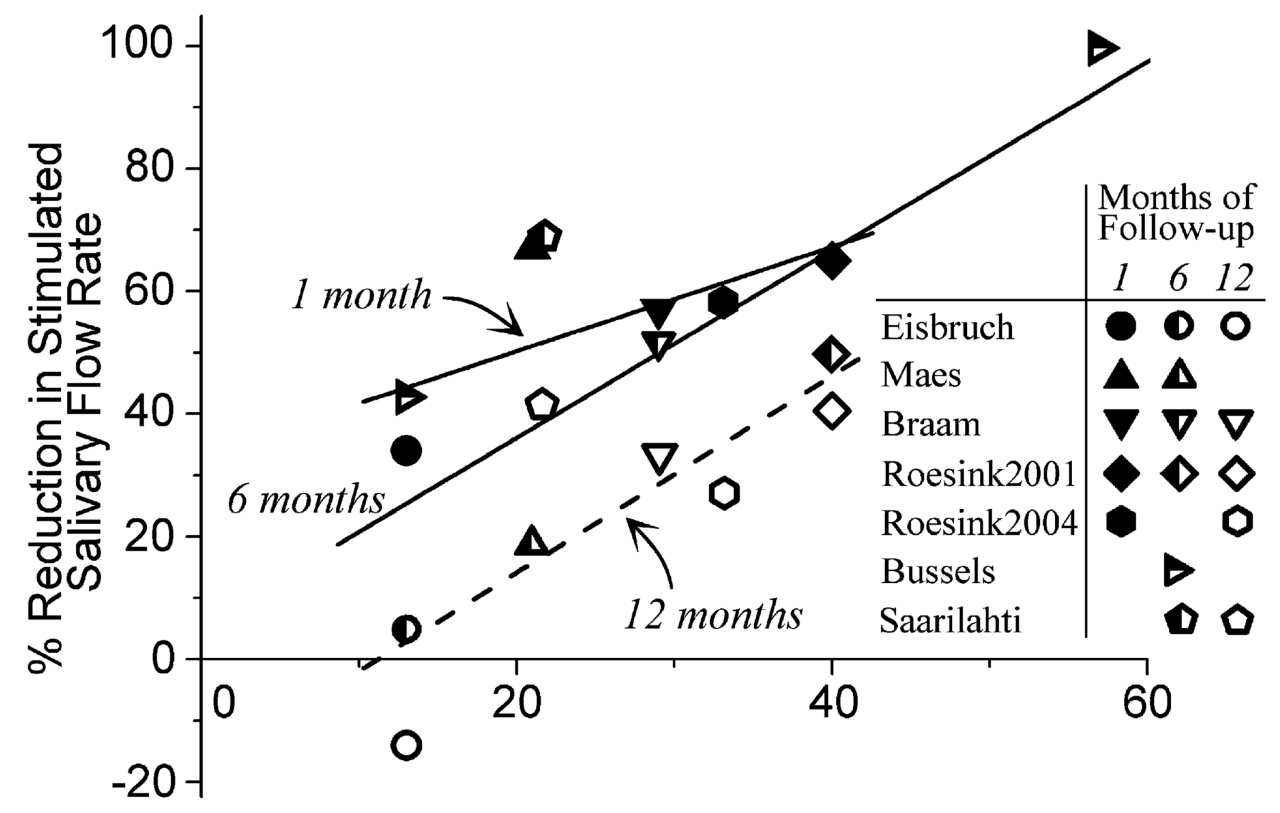

\section{Mean Parotid Gland Dose (Gy)}

Fig. 2.

Mean percentage of reduction in stimulated salivary flow rate vs. mean parotid gland dose for different follow-up durations $(8,10,12,14,15,16)$. Follow-up durations of 1,6 , and 12 months represent ranges of 1-1.5, 6-7, and 12 months, respectively. Linear fits of data from different follow-up intervals shown. Dose-response effect appears present at all times, with shift of data to right with time, suggesting functional repair or regeneration. 


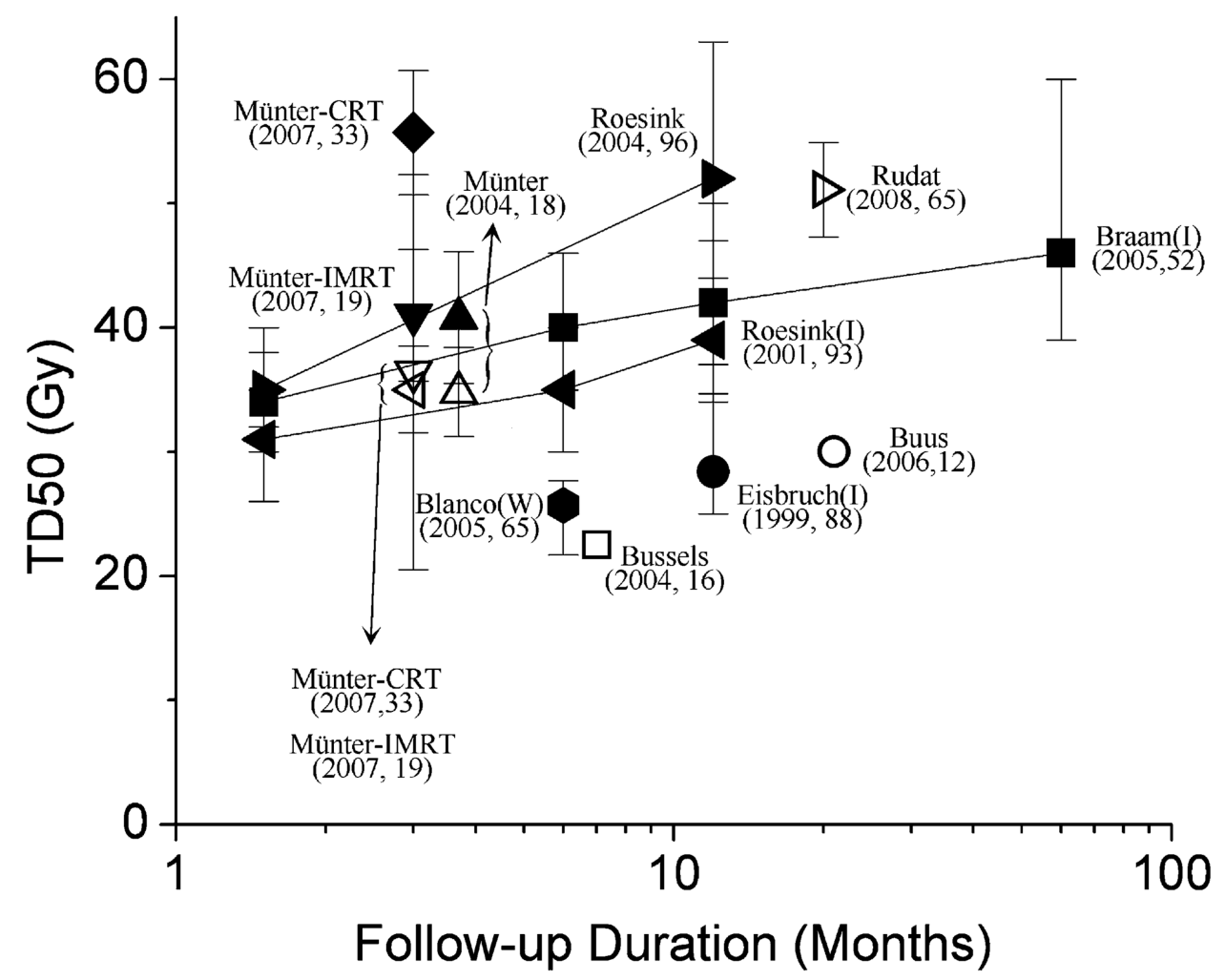

Fig. 3.

Reported tissue dose required for $50 \%$ response for loss of stimulated saliva flow after radiotherapy $(\mathrm{RT})(2,6,10,14-20)$ for single parotid gland. Endpoint considered in reports was salivary flow reduction to $<25 \%$ (black symbols) or $<50 \%$ (gray symbols) of pretreatment value. Tissue dose required for $50 \%$ response defined as dose at which $50 \%$ of patients developed complications. Error bars (if shown) indicate 95\% confidence intervals; refer to original publications for exact meaning. 95\% Confidence intervals for studies by Munter et al. $(19,20)$ were estimated from standard errors provided. Lines connect points from data sets with measurements taken at more than one interval after radiotherapy. Most studies used salivary gland scintigraphy. Some studies measured physical production (ipsilateral salivary flow or whole salivary flow; marked with "I" or "W", respectively). Data from Buus et al. (2) (which did not include preradiotherapy assessments) derived by comparing different regions of parotid gland that had received different doses. Each label gives number of patients. Note, most imaging-derived endpoint data had greater values for tissue dose required for $50 \%$ response (TD50) than measured salivary data. CRT $=$ conformal radiotherapy; IMRT = intensity-modulated radiotherapy. 


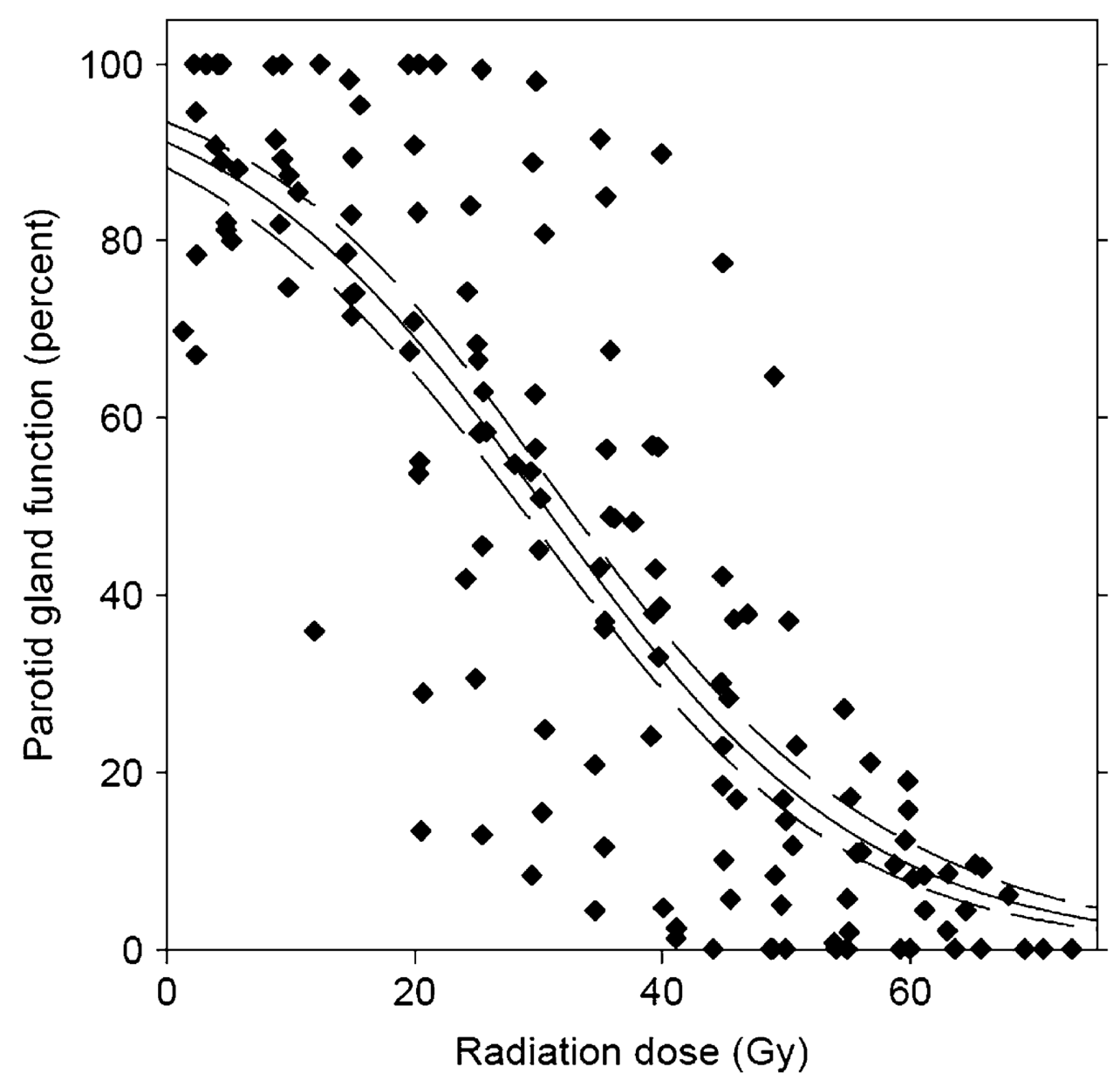

Fig. 4.

Population-based dose vs. local function response (salivary function at rest) from imaging study by Buus et al. (2). Local functional decline in metabolic clearance of parotid salivary glands vs. local dose, according to voxel-by-voxel estimated time-activity curves of intravenously injected C11-methionine. Data points from 12 patients shown, along with best-fit curve and $95 \%$ confidence intervals of curve fit. Individual gland curves reported by Buus et al. (2) deviated significantly from this population average curve (reproduced from Buus et al. [2], used with permission.) This population curve demonstrated functional decline in salivary function even at low doses. 


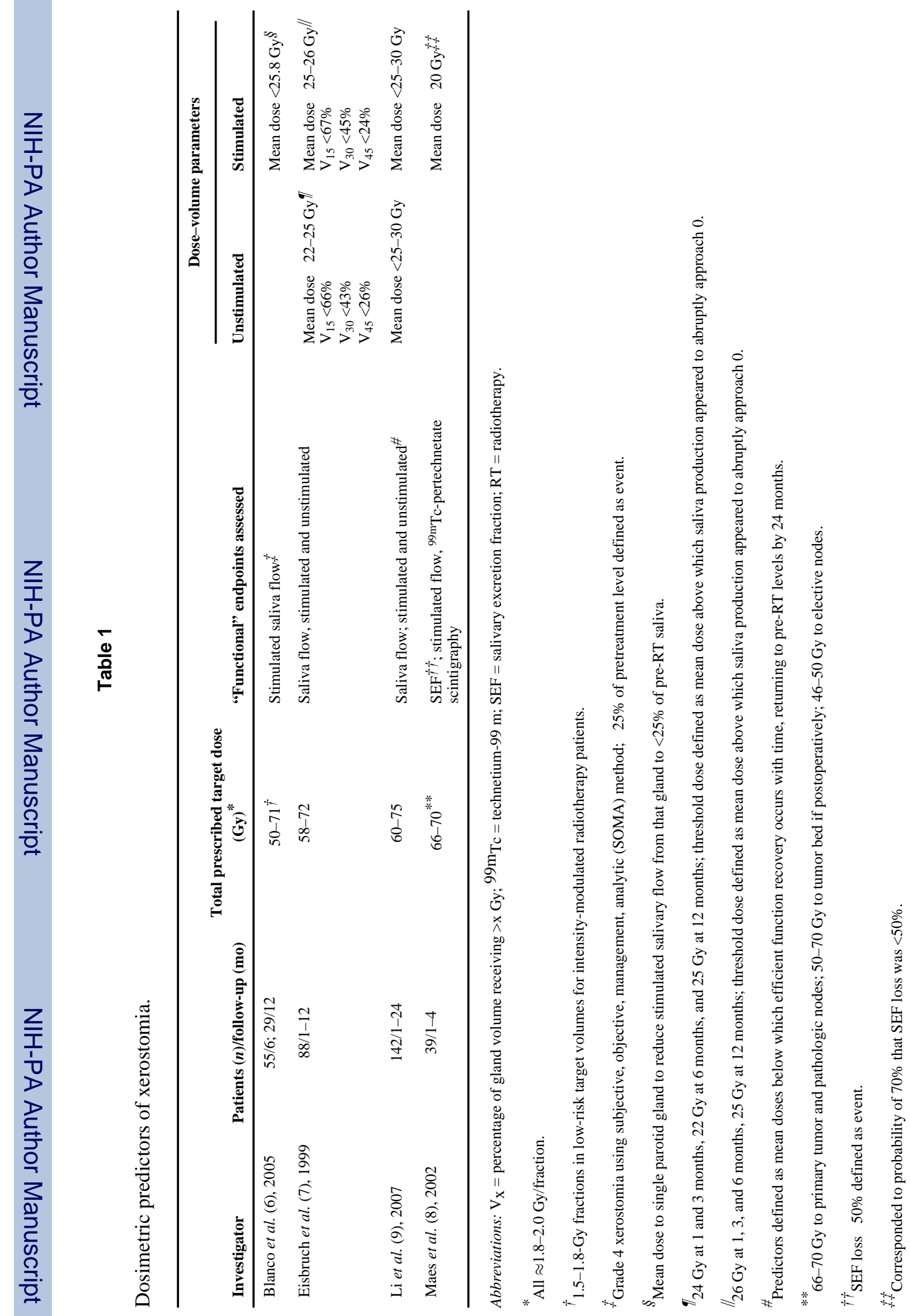

